\title{
A tutorial on the use of informative Bayesian priors for minority research
}

Authors:

Taylor Winter, Department of Psychology, Victoria University of Wellington, Wellington, New Zealand.

Benjamin C. Riordan, Discipline of Addiction Medicine, Central Clinical School, Faculty of Medicine and Health, University of Sydney, Sydney, Australia.

Anthony Surace, Department of Behavioural and Social Sciences, Center for Alcohol and Addiction Studies, Brown University School of Public Health, Providence, Rhode Island, U.S.A.

Damian Scarf, Department of Psychology, University of Otago, Dunedin, New Zealand

Paul E. Jose, Department of Psychology, Victoria University of Wellington, Wellington, New Zealand.

Running head: Bayesian priors for research with minority groups

Word count: 3195

Declaration of conflicts: None

Acknowledgement: We wish to acknowledge Gareth Minshall who provided feedback on an initial draft of this manuscript.

Corresponding Author: Taylor Winter - taylor.winter@vuw.ac.nz 
RUNNING HEAD: Bayesian priors for research with minority groups

\begin{abstract}
Aims. Quantifying differences between minority and majority groups, such as sexual minorities (SM) and heterosexuals, is difficult due to small sample sizes. Bayesian analyses is one solution to addressing small sample sizes in minority group research, whereby previous research can be used to inform our models. In the present tutorial, we offered an overview of Bayesian statistics and described an approach to constructing informed priors using a large survey when estimating values in a smaller survey. In an applied example, we determined whether SMs in New Zealand reported more stress relative to heterosexuals and whether stress mediates the link between SM status and alcohol use.
\end{abstract}

Design. Two cross-sectional, stratified, and nationally representative health surveys from the US (National Survey of Drug Use and Health (NSDUH)) and New Zealand (New Zealand Health Survey (NZHS)).

Settings. United States, New Zealand

Participants. We used data from 83,661 $(\mathrm{SMs}=5593)$ survey respondents in the US and 24,098 respondents in NZ $(\mathrm{SMs}=619)$.

Measurements. Demographic items (sex, age, ethnicity, sexual identity), the Kessler psychological distress scale, and the Alcohol Use Disorder Identification Test (AUDIT).

Findings. Using a larger survey to inform priors reduced the uncertainty of estimates derived from small subgroups in a smaller survey relative to uninformed priors.

Conclusion. Informed Bayesian analyses are an important tool for researchers studying minority groups and the application of informative priors allows for more reliable estimates of health disparities.

Key Words: Bayesian statistics, Alcohol, minority stress, health disparities 
RUNNING HEAD: Bayesian priors for research with minority groups

\section{A tutorial on the use of informative Bayesian priors for substance research with minority groups}

In the ongoing debate about whether Bayesian statistics offer advantages over classical statistics, it is useful to highlight specific ways in which a Bayesian approach confers unique advantages over classical analytic approaches. In the present article, we demonstrate how Bayesian statistics, specifically the use of informed priors, are an important tool for minority group substance use research. For example, sexual minority populations (i.e. those whose sexual identity, orientation, or practices differ from most of the surrounding society, $[\mathrm{SM}]$ ) consistently show elevated rates of substance use and related health problems relative to heterosexual populations (Dermody et al., 2013). Interpreting such findings and explanatory factors can be difficult given the relative size of such populations. In other words, we need to obtain reliable statistics on minority groups due to their over-representation in measures of illhealth, but this is difficult due to the relative rarity of minority groups in the general population. Indeed, there has been a "call to action" for future research to isolate the drivers of these disparities (Zemore et al., 2018) and informed Bayesian analyses may play a key role in producing more accurate estimates in groups with typically small sample sizes. In the present study, we aim to provide an example method using a Bayesian approach to apply a 'prior' (i.e., a prior probability distribution based on previous empirical research) from the National Survey of Drug Use and Health (NSDUH), to a smaller survey, the New Zealand Health Survey (NZHS). To supplement the analysis, we include all our code and example datasets that were generated to resemble the data that is not publicly available.

\section{The example problem: SM research from the NZHS}

In our preliminary research, we have published evidence of the increased prevalence of hazardous drinking in the New Zealand SM population (Surace et al., 2019). Surace et al. 
RUNNING HEAD: Bayesian priors for research with minority groups

(2019) used the 2016 and 2017 NZHS, a nationally representative survey of approximately 10,000 individuals each year to investigate the increased risk of hazardous drinking among SMs. However, even when pooling two years of data, only 626 people identified as SMs (i.e., homosexual or bisexual). In order to preserve analytic power, we were forced to pool these unique populations (i.e. homosexual and bisexual identified participants) into a single SM group. The study found that there was an overall increase in hazardous drinking for SMs, but this effect appeared to be moderated by gender. Specifically, SM women appeared to have higher risk of hazardous drinking than SM men. However, the limited sample size meant we were unable to (1) examine how hazardous alcohol use varied between SM subgroups, (i.e., homosexual, bisexual), and (2) evaluate gender as a moderator of individual SM subgroups. This approach is especially problematic given that subpopulations within the larger SM community display unique health disparities relative to heterosexuals and other SMs (Fish et al., 2017).

\section{Overview of Bayesian statistics}

Bayesian inference combines our prior knowledge concerning the probability of an effect (i.e., stress experienced by SM in communities around the world) with the observed probability of an effect in our data (i.e., stress experienced by the New Zealand SM community). Under Bayesian inference, a prior probability distribution is specified for each model parameter. This distribution is then updated using evidence found in the new data (the likelihood) to generate a posterior probability distribution. In other words, a posterior distribution for a model parameter is proportional to the product of the prior distribution and the observed likelihood (Equation 1). We can then use the posterior distribution for statistical inference such as prediction intervals or probability estimates for a hypothesised effect. One point of contrast, however, is that in a Bayesian framework we estimate the probability of an 
RUNNING HEAD: Bayesian priors for research with minority groups

effect, whereas in classical statistics we estimate the probability of our data relative to our hypothesis of an effect.

Equation 1. Relationship between prior, likelihood, and posterior probability distributions. A normalising constant is used to limit the posterior distribution from summing to a probability greater than one (which would be mathematically implausible).

$$
\text { Posterior }=\frac{\text { Likelihood } \times \text { Prior }}{\text { Normalising constant }}
$$

\section{An example of priors}

An important part of Bayesian analysis is determining how to specify the prior. Priors vary in their 'informativeness' (i.e., how much they stipulate variance and mean level information). If a prior is non-informative, it is a distribution that covers a large range of credible values for an effect (e.g., values between the minimum and maximum values are equally likely; see Figure 1a). Non-informative priors result in a posterior distribution similar to the likelihood and therefore give similar results to classical analyses. Informative priors have a narrower range of credible values and therefore more strongly constrain the posterior distribution (see Figure 1b). The use of informative priors within Bayesian estimation provides an avenue for updating existing knowledge with new data. This approach can be particularly useful when small sample sizes do not allow independent analysis, but substantial knowledge already exists from previous studies using different data sources on a similar population.

For instance, when estimating average population height, we would note that on average studies report a mean height of $160 \mathrm{~cm}(\mu)$, and reported means tend to vary with a standard deviation of $20 \mathrm{~cm}\left(\sigma_{\mu}\right)$. If we draw a new, albeit small sample $(\mathrm{n}=50)$ from the total population and observe a mean height of $\bar{x}=120 \mathrm{~cm}$ and $s=40 \mathrm{~cm}$ (i.e., observed likelihood), we can combine our prior information $\left(\mu=160 \mathrm{~cm}\right.$ and $\left.\sigma_{\mu}=20 \mathrm{~cm}\right)$ with our observed likelihood 
RUNNING HEAD: Bayesian priors for research with minority groups

using Bayes' theorem to get a new probability distribution for our population mean. As the prior becomes more informative (i.e., variance of the mean decreases), the posterior distribution is more likely to resemble the prior (Figure 1c). In the case of non-informative priors (i.e., very high variance) the posterior distribution takes on the form of the observed likelihood in the data (Figure 1a). Similarly, when we have a very large sample or low sample variance, the prior has little influence on the posterior distribution relative to the observed likelihood.

This overview of Bayesian statistics is brief, but it outlines how we can use a prior distribution to inform our observed likelihood, and to produce a posterior distribution with lower variance and more representative population estimates (for a more exhaustive overview, see Etz and Vandekerckhove, 2017; Kruschke, 2015; McElreath, 2018).

Figure 1. Prior, likelihood, and posterior distributions for the example of mean height with weakly informative (1a), moderately informative (1b), and highly informative (1c) priors

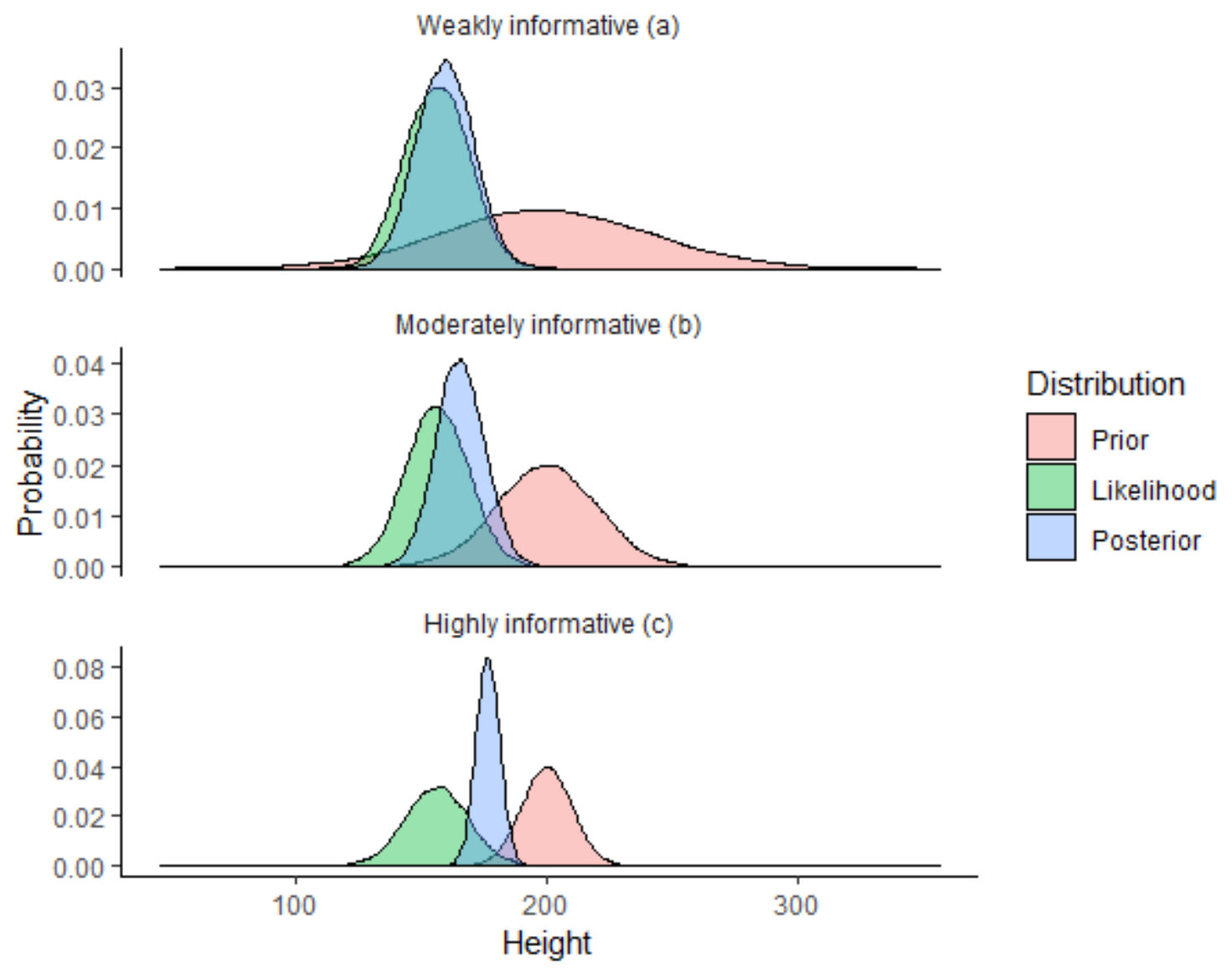


RUNNING HEAD: Bayesian priors for research with minority groups

\section{The present example}

In the present example, we used data from the 2016 and 2017 National Survey of Drug Use and Health (NSDUH; $n=83,661$ ) to construct our prior (aim 1) and subsequently used it to estimate similar effects in our New Zealand sample - the pooled 2016-17 NZHS ( $\mathrm{n}=26,218)$ (aim 2). We then applied the same priors to a simple mediation analysis, illustrating that information for only one component of a path analysis can substantially increase the overall statistical power of a model (aim 3). We hypothesised that SMs (i.e., homosexuals and bisexuals) would report higher levels of stress than heterosexuals. We also hypothesised that female SMs would evidence higher odds of stress due to SM status relative to SM males (i.e., a moderating effect of gender). Lastly, we hypothesised that stress would explain an increased risk of hazardous drinking observed in SMs. All code, synthetic datasets, and an exhaustive walkthrough has been provided on GitHub and can be found at the following link: https://haututu.github.io/bayesianPriorTutorial/.

\section{Method}

\section{NSDUH}

The NSDUH is an annual survey collected in face-to-face interviews from a stratified sample throughout the United States. The sample has a target response of 67,504 individuals aged twelve years or older. Data are publicly available and can be found at https://www.samhsa.gov/data/. We have also generated a sample dataset which can produce similar effects but is substantially smaller, i.e., it has more palatable runtime during analysis, (see GitHub repository).

\section{NZHS}

The NZHS is an annual survey collected with a combination of face-to-face and computer-assisted interviews in a stratified sample of adults aged 15 and over throughout 
RUNNING HEAD: Bayesian priors for research with minority groups

New Zealand. Confidential survey records can be accessed for research purposes via an application to Stats NZ. The survey covers a very broad range of health domains and behaviours. More information on sample design and content can be found at https://www.health.govt.nz/nz-health-statistics. Although the data is not publicly available, we include a mock dataset which has similar properties (see see GitHub repository).

\section{Demographics and measures}

NSDUH. We pooled participants across 2016 and 2017 to attain a sample of 113,173 participants. All participants at or below age 16 years old did not complete the stress questions, leaving a final sample of 83,661 participants in the NSDUH who completed all questions in the analysis, of which 44,424 self-identified as female. To maintain confidentiality, the NSDUH decreases the granularity of some measures such as age. In the NSDUH, age is grouped by single year until age 21 , two-year age groups until age 25 , fiveyear age groups until age 34, 15-year age groups until 65, and lastly 65 and older. Participants who refused to answer or indicated 'other' sexual identity, were removed from the analyses. See Table 1 for additional demographics.

Table 1. Summary of NSDUH Demographic Data Stratified by Sexual Orientation.

\begin{tabular}{lcccc}
\hline & Heterosexual & Homosexual & Bisexual & Total \\
\hline $\mathrm{N}$ & 78068 & 1831 & 3762 & 83661 \\
Females & $52.0 \%$ & $47.6 \%$ & $77.6 \%$ & $53.1 \%$ \\
Median age group & $30-34$ & $26-29$ & $24-25$ & $30-34$ \\
Median income group (\$000s) & $40-50$ & $40-50$ & $30-40$ & $40-50$ \\
High distress & $6.8 \%$ & $17.7 \%$ & $25.5 \%$ & $7.9 \%$ \\
\hline
\end{tabular}

We used household income as a general indication of a participant's socio-economic status. Income was also banded to maintain confidentiality in $\$ 10,000$ increments up to $\$ 50,000$, then $\$ 50 \mathrm{k}$ to $\$ 75 \mathrm{k}$, and $\$ 75 \mathrm{k}$ or more. We considered this measure to be approximately equivalent to the New Zealand Deprivation index (NZDep). 
RUNNING HEAD: Bayesian priors for research with minority groups

Stress was measured using the Kessler Psychological Distress Scale - short version (K6). This version of the Kessler scale asks six questions about feelings of distress in the last month. A score over 12 (out of a maximum 24) is considered high distress and indicative of risk of mental health issues, which we used as a binary outcome in the present study.

NZHS. There were 24,098 participants in the NZHS, 13,735 were female and ages ranged from 18 to 90 years old $(\mu=50$ years old). The NZHS did not require measures to maintain confidentiality so we used single year age. The sample was pooled across two survey years, 2016 and 2017, and invariance testing supported no systematic differences between the two years. The 7\% of the total sample who reported they 'did not know' their sexual orientation or refused to answer were subsequently removed from analyses. See Table 2 for additional demographics.

Table 2. Summary of NZHS Demographic Data Stratified by Sexual Orientation.

\begin{tabular}{lcccc}
\hline & Heterosexual & Homosexual & Bisexual & Total \\
\hline $\mathrm{N}$ & 23479 & 280 & 339 & 24098 \\
Females & $56.8 \%$ & $47.9 \%$ & $70.2 \%$ & $56.9 \%$ \\
Mean age & 50 & 43 & 34 & 50 \\
Median NZDep & 6 & 7 & 7 & 6 \\
High distress & $7.5 \%$ & $12.9 \%$ & $23.0 \%$ & $7.8 \%$ \\
Hazardous drinking & $19.7 \%$ & $30.5 \%$ & $35.0 \%$ & $20.0 \%$ \\
\hline
\end{tabular}

Note. NZDep is the New Zealand deprivation score.

The New Zealand Deprivation Index (NZDep) is a decile index applied to the smallest defined geographies in New Zealand (containing an average of 100 households). The index is derived from a range of socioeconomic variables gathered in the New Zealand Census and it has been demonstrated to be a good measure of the socioeconomic status of a household (Atkinson et al., 2014).

Stress in the NZHS was measured using the Kessler Psychological Distress Scale long version (K10). It contains 10 questions about distress in the last month and we binarized the measure using a cut-off of 11 or higher out of 54. Analyses showed that despite the two 
RUNNING HEAD: Bayesian priors for research with minority groups

cut-offs being different between $\mathrm{K} 6$ and $\mathrm{K} 10$, the high stressed populations were still relatively the same size and fit for comparison between survey sources.

Hazardous drinking was measured using the Alcohol Use Disorder Identification Test (AUDIT; Bohn et al., 1995). The AUDIT contains 10 questions and is a tool used to screen for alcohol use disorders. A score 8 or greater (out of 40) indicates a pattern of drinking which is considered hazardous and we binarized the measure using a cut-off of eight points.

\section{Analytical approach}

In order to model stress for the NSDUH (aim 1), stress for the NZHS (aims 2 and 3), and hazardous drinking for the NZHS (aim 3), we used Bayesian logistic regression implemented in R v3.4.4 using the brms v2.7.0 package (Bürkner, 2017; R Core Team, 2019). The brms package facilitates simple ways of implementing a broad range of regression and multi-level regression models in $\mathrm{R}$ using a statistical language called Stan (Carpenter et al., 2017). Mediation analysis was conducted using brms using the product of coefficients approach.

NSDUH. In the US data, age and household income were banded and therefore we accounted for the ordinal nature by modelling monotonic effects. In short, this approach involves modelling a variable as a continuous variable to obtain a coefficient for the trend, then modelling how much each category in the variable deviates from the trend. For the purposes of informing our New Zealand study, we ignored the deviations and used the trend term to inform our continuous variables in the NZHS. Pertaining to our hypotheses, we modelled sexual identity and gender, and the interaction of the two variables, to test if gender was a moderator of sexual identity on risk of high stress.

NZHS. The NZHS data did not have banded variables, so we were able to add age and NZDep as continuous covariates in the model. Like the US study, we added sexual identity, 
RUNNING HEAD: Bayesian priors for research with minority groups

gender, and their interaction to test our hypotheses that: 1) sexual identity would be associated with an increase in stress, and 2) gender (i.e., females) would exacerbate the effect of sexual identity on stress. We then conducted a simple mediation analysis with hazardous drinking as the outcome, stress as a mediator, and sexual identity as the treatment variable (see Figure 2).

Figure 2. Simple mediation between SM status and hazardous drinking with discrimination as a mediator.

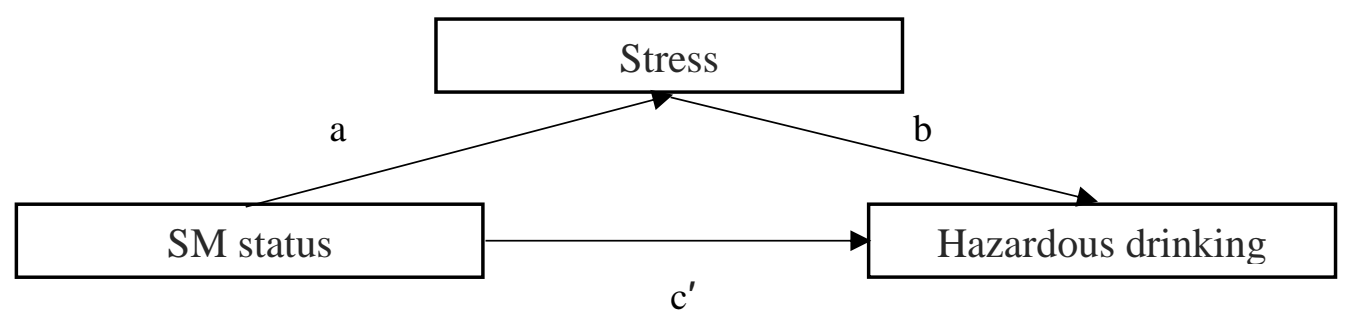

Construction of priors. We used normal distributions to represent the prior probability of each effect in our regression analyses of the NZ data. The mean and standard deviation for the prior of each effect was stipulated to be the coefficient and estimated error for each effect in the US data. In order to integrate our view that New Zealand may have different effect sizes to the US, we doubled the standard deviation of each prior to indicate a broader range of likely effect sizes in the NZ data. In the case of age and NZDep (the latter using the coefficient of household income), we were uncertain that trends from banded coefficients in the US data would offer valuable information and therefore inflated standard deviations of priors by a factor of three.

\section{Results}

Did SM groups report higher levels of stress than heterosexuals in the NSDUH? And if so, was this relationship moderated by sex?

Our analyses yielded a more than a $99 \%$ probability that SM status was associated with higher likelihood of stress (hypothesis one). The odds of reporting stress were 2.8 and 3.3 times 
RUNNING HEAD: Bayesian priors for research with minority groups

higher than heterosexuals for homosexuals and bisexuals respectively, with bisexuals also being at significantly higher odds than homosexuals with 95\% probability (Table 3). We did not, however, find support for our second hypothesis of an interaction between sex and SM status, both of which yielded less than a $70 \%$ probability. The estimates and error terms in Table 3 were then used to construct priors for the New Zealand data.

Table 3. Odds of being highly stressed (using US data)

\begin{tabular}{lccc}
\hline & Estimate & Error & Probability \\
\hline Intercept & 0.19 & 1.06 & $>99.99 \%$ \\
Sex & 1.34 & 1.03 & $>99.99 \%$ \\
Homosexual & 2.80 & 1.10 & $>99.99 \%$ \\
Bisexual & 3.27 & 1.10 & $>99.99 \%$ \\
Sex * Homosexual & 0.94 & 1.14 & $69.15 \%$ \\
Sex * Bisexual & 0.99 & 1.11 & $53.03 \%$ \\
Age & 0.14 & 1.10 & $>99.99 \%$ \\
Income & 0.40 & 1.04 & $>99.99 \%$ \\
\hline
\end{tabular}

\section{Did SM groups report higher levels of stress than heterosexuals (data from NZHS, using NSDUH for priors)?}

We found more than a $99 \%$ probability that SM status would increase the odds of stress in comparison to heterosexuals, and the odds appeared to be higher for bisexuals than homosexuals (2.9 and 2.2 increase in odds, respectively). Similar to the US data, the findings did not support sex as a moderator. In Table 4, results of the model using weakly informative priors are labelled informed whereas results of a model using non-informative priors, i.e., flat priors, are labelled non-informed. In this case the non-informed model is analogous to the results from a frequentist (i.e., classical) logistic regression.

Table 4. Odds of reporting high levels of stress (using New Zealand data)

\begin{tabular}{|c|c|c|c|c|c|}
\hline & \multicolumn{2}{|c|}{ Coefficient } & \multicolumn{2}{|c|}{ Error } & \\
\hline & Informed & $\begin{array}{c}\text { Non- } \\
\text { informed }\end{array}$ & Informed & $\begin{array}{c}\text { Non- } \\
\text { informed }\end{array}$ & $\begin{array}{l}\text { Probability } \\
(\text { Odds }>1)\end{array}$ \\
\hline Intercept & 0.07 & 0.07 & 1.11 & 1.10 & $>99.99 \%$ \\
\hline
\end{tabular}


RUNNING HEAD: Bayesian priors for research with minority groups

\begin{tabular}{lccccc} 
age & 0.99 & 0.99 & 1 & 1.00 & $>99.99 \%$ \\
Sex (Male) & 0.69 & 0.68 & 1.05 & 1.05 & $>99.99 \%$ \\
Homosexual & 2.22 & 1.58 & 1.14 & 1.29 & $>99.99 \%$ \\
Bisexual & 2.93 & 2.74 & 1.12 & 1.17 & $>99.99 \%$ \\
NZDep & 1.16 & 1.16 & 1.01 & 1.01 & $>99.99 \%$ \\
Sex*Homosexual & 0.88 & 1.11 & 1.19 & 1.45 & $77.30 \%$ \\
Sex*Bisexual & 0.93 & 0.90 & 1.18 & 1.37 & $65.75 \%$ \\
\hline
\end{tabular}

Our priors from the NSDUH appeared to be realistic in that the posterior distributions seemed to significantly overlap with the prior distributions (Figure 3). Little overlap of distributions or convergence issues are noted if priors are inappropriate. The prior for the influence of SM status on stress increased the odds ratio of being stressed from 1.58 to 2.22 . In contrast, the odds ratio for bisexuals remained about the same between non-informed and informed analyses. In both cases, homosexual and bisexual effects on stress yielded slightly reduced error due to the use of informed priors.

Figure 3. Comparison of prior distributions (U.S.) with posterior distributions for homosexuals and bisexuals (New Zealand).

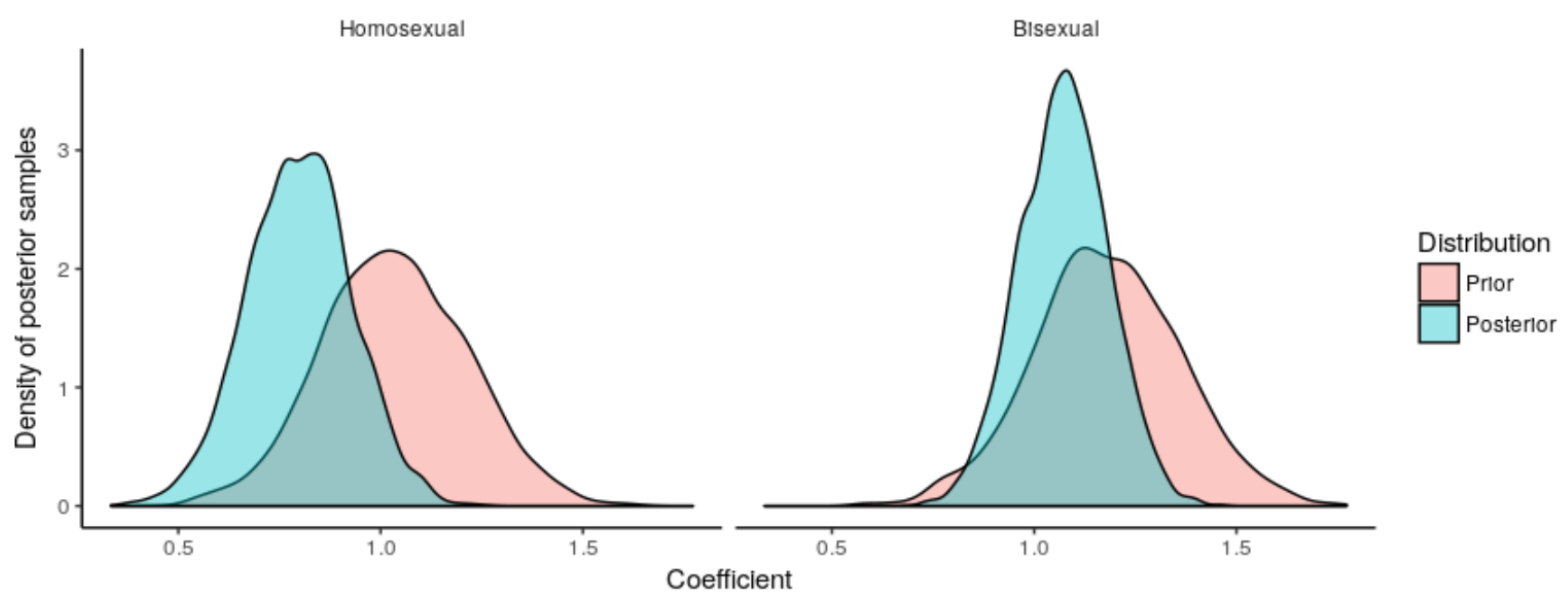

Did higher levels of stress explain greater risk of hazardous drinking in SMs (data from NZHS, using NSDUH for priors).

Using the Sobel product of coefficients approach (a times b, see Jose, 2013), we found that $48 \%$ [95\% CI $=31 \%, 66 \%$ ] of the increase in odds of hazardous drinking associated with 
RUNNING HEAD: Bayesian priors for research with minority groups

homosexuals could be explained by the mediation of high stress (Figure 4). Similarly, we found that $49 \%$ [95\% CI $=37 \%, 62 \%]$ of the increased risk of bisexuals being hazardous drinkers could be explained by the mediation of high stress. These results supported our hypothesis that SMs could be at higher risk of poor health outcomes such as hazardous drinking than heterosexuals due to the mediating role of increased stress.

Figure 4. Comparison of the proportion of an SM effect on risk of drinking that can be explained by stress between models with informative priors and naïve priors. Error bars are the $95 \%$ credible intervals, that is, an interval that is $95 \%$ likely to contain the true effect.

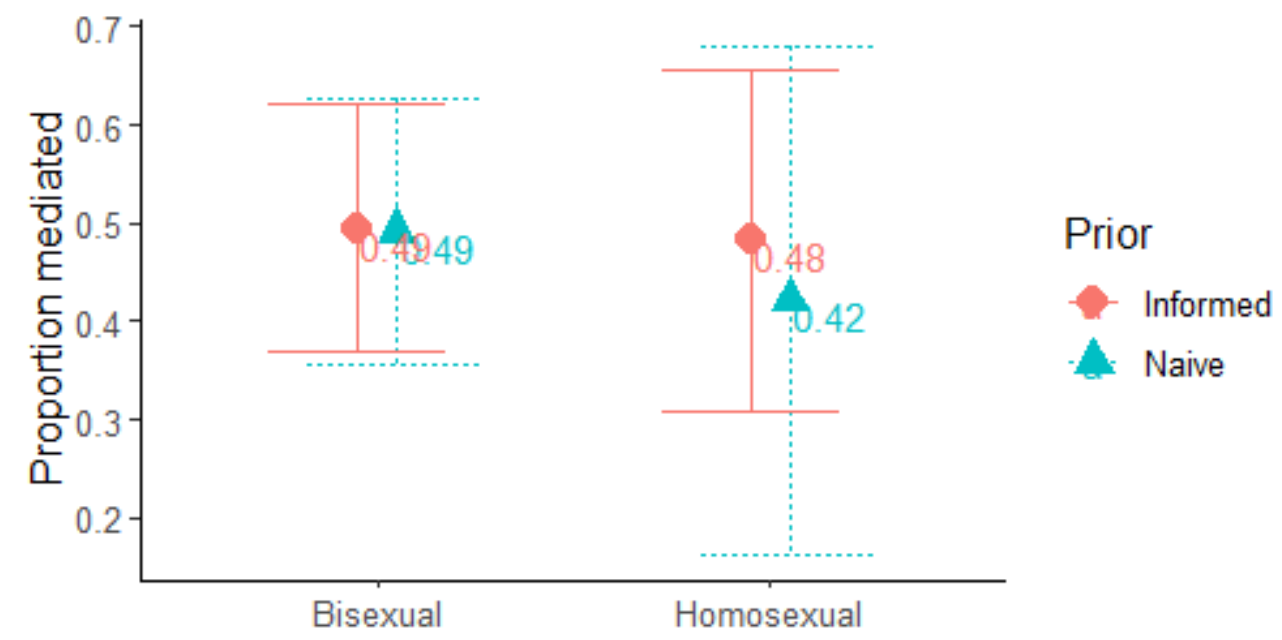

Discussion

The chief goal of this manuscript was to present a brief introduction to Bayesian statistics and demonstrate how informed priors may be applied to research with small groups within larger populations. Our study demonstrated that informative priors produced an observable reduction in uncertainty of estimates derived from small subgroups relative to uninformed priors (which yield similar results to a classical approach). We also demonstrated the utility of informative priors in structural equation models (i.e., the mediation model tested here). Specifically, we implemented an informed prior for the relationship between SM status and stress, which then reduced the overall uncertainty in our mediation effect. In future work, 
RUNNING HEAD: Bayesian priors for research with minority groups

we recommend that researchers consider leveraging trends noted in large samples to smaller samples, and this approach can be generalised to numerous statistical applications beyond the specific ones demonstrated here.

Datasets, both large and small, and expert opinion can be used to better determine the level of informativeness in priors. In our case, we erred on the conservative side and inflated error terms in the US data by a factor of two, but instead we could have lowered the mean of the SM coefficients based on evidence from a smaller NZ-based survey, the General Social Survey, and used a more informative prior rather than inflating error terms from the US data. In best practice, it is not uncommon to use expert opinion, or similar small samples in previous research to produce more accurate informed priors (Veen et al., 2017). However, in the absence of robust previous research, it becomes more important to properly investigate the effect of the priors on your observed estimates. One simple approach is to conduct a sensitivity analyses using a variety of levels of informativeness and different orders of magnitude in the mean of the prior to determine what effect, and how great an effect, the prior is having on your observed values (Kruschke, 2015). There are also a number of other approaches to consider when constructing and validating priors that were beyond the scope of this study but we highly encourage readers to check the following resource by Gelman (2019).

The present study offered an introductory explanation of priors within a Bayesian framework and discussed how and what it means to use an informative prior. Our approach leveraged information from a large previously collected study and applied this information to a relatively small NZ survey. We demonstrated that the uncertainty around risks of stress for SM groups can be observably reduced when leveraging off larger previously collected studies. Readers are encouraged to consider how they may construct priors when conducting a Bayesian analysis to take advantage of this useful strategy. 
RUNNING HEAD: Bayesian priors for research with minority groups 
RUNNING HEAD: Bayesian priors for research with minority groups

\section{References}

Atkinson J, Salmond C and Crampton P (2014) NZDep2013 Index of Deprivation.: 64.

Bohn MJ, Babor TF and Kranzler HR (1995) The Alcohol Use Disorders Identification Test (AUDIT): validation of a screening instrument for use in medical settings. Journal of Studies on Alcohol 56(4). Alcohol Research Documentation, Inc.: 423-432. DOI: 10.15288/jsa.1995.56.423.

Bürkner P-C (2017) brms: An R Package for Bayesian Multilevel Models Using Stan. Journal of Statistical Software 80(1): 1-28. DOI: 10.18637/jss.v080.i01.

Carpenter B, Gelman A, Hoffman MD, et al. (2017) Stan: A Probabilistic Programming Language. Journal of Statistical Software 76(1). 1: 1-32. DOI: 10.18637/jss.v076.i01.

Dermody SS, Marshal MP, Cheong J, et al. (2013) Longitudinal Disparities of Hazardous Drinking Between Sexual Minority and Heterosexual Individuals from Adolescence to Young Adulthood. Journal of Youth and Adolescence 43(1). Springer Science and Business Media LLC: 30-39. DOI: 10.1007/s10964-013-9905-9.

Etz A and Vandekerckhove J (2017) Introduction to Bayesian Inference for Psychology. Psychonomic Bulletin \& Review 25(1). Springer Science and Business Media LLC: 534. DOI: 10.3758/s13423-017-1262-3.

Fish JN, Hughes TL and Russell ST (2017) Sexual identity differences in high-intensity binge drinking: findings from a US national sample. Addiction 113(4). Wiley: 749-758. DOI: $10.1111 /$ add.14041. 
RUNNING HEAD: Bayesian priors for research with minority groups

Gelman A (2019) Prior Choice Recommendations. In: Prior Choice Recommendations. Available at: https://github.com/stan-dev/stan/wiki/Prior-Choice-Recommendations (accessed 4 November 2019).

Kruschke JK (2015) Doing Bayesian Data Analysis. In: Doing Bayesian Data Analysis. Elsevier, pp. 193-219. DOI: 10.1016/b978-0-12-405888-0.00008-8.

McElreath R (2018) Statistical Rethinking. Chapman and Hall/CRC. DOI: $10.1201 / 9781315372495$

R Core Team (2019) R: A Language and Environment for Statistical Computing. Vienna, Austria: R Foundation for Statistical Computing. Available at: https://www.Rproject.org/.

Surace A, Riordan B and Winter T (2019) Do New Zealand sexual minorities engage in more hazardous drinking than non-sexual minorities? Drug and Alcohol Review.

Veen D, Stoel D, Zondervan-Zwijnenburg M, et al. (2017) Proposal for a Five-Step Method to Elicit Expert Judgment. Frontiers in Psychology 8. Frontiers Media SA. DOI: 10.3389/fpsyg.2017.02110.

Zemore SE, Karriker-Jaffe KJ, Mulia N, et al. (2018) The Future of Research on AlcoholRelated Disparities Across U.S. Racial/Ethnic Groups: A Plan of Attack. Journal of Studies on Alcohol and Drugs 79(1). Alcohol Research Documentation, Inc.: 7-21. DOI: $10.15288 /$ jsad.2018.79.7. 